STRUCTURAL BIOLOGY COMMUNICATIONS

ISSN 2053-230X

Received 9 October 2017

Accepted 13 December 2017

Edited by I. Tanaka, Hokkaido University, Japan

‡ These authors made equal contributions.

Keywords: decarboxylases; pyridoxal phosphate; catalysis; conformational change.

PDB reference: mouse GADL1, 6enz

Supporting information: this article has supporting information at journals.iucr.org/f

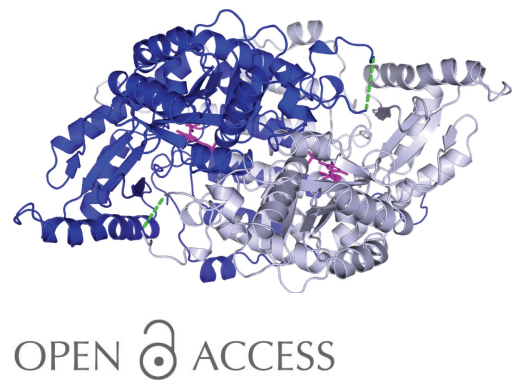

\section{Structure of the mouse acidic amino acid decarboxylase GADL1}

\author{
Arne Raasakka, ${ }^{\mathrm{a}} \neq$ Elaheh Mahootchi, ${ }^{\mathrm{a}, \mathrm{b}} \neq$ Ingeborg Winge, ${ }^{\mathrm{a}, \mathrm{b}}$ Weisha Luan, \\ Petri Kursula $^{\mathrm{a}, \mathrm{c} *}$ and Jan Haavik ${ }^{\mathrm{a}, \mathrm{b}, \mathrm{d} *}$
}

a'Department of Biomedicine, University of Bergen, Jonas Lies Vei 91, 5009 Bergen, Norway, ${ }^{\mathbf{b}}$ K. G. Jebsen Centre for
Research on Neuropsychiatric Disorders, University of Bergen, Jonas Lies Vei 91, 5009 Bergen, Norway, ${ }^{\mathbf{F}}$ Faculty of
Biochemistry and Molecular Medicine, University of Oulu, PO Box 5400, 90014 Oulu, Finland, and ${ }^{\mathbf{d}}$ Division of
Psychiatry, Haukeland University Hospital, Bergen, Norway. *Correspondence e-mail: petri.kursula@uib.no,
jan.haavik@uib.no

Pyridoxal 5'-phosphate (PLP) is a ubiquitous cofactor in various enzyme classes, including PLP-dependent decarboxylases. A recently discovered member of this class is glutamic acid decarboxylase-like protein 1 (GADL1), which lacks the activity to decarboxylate glutamate to $\gamma$-aminobutyrate, despite its homology to glutamic acid decarboxylase. Among the acidic amino acid decarboxylases, GADL1 is most similar to cysteine sulfinic acid decarboxylase (CSAD), but the physiological function of GADL1 is unclear, although its expression pattern and activity suggest a role in neurotransmitter and neuroprotectant metabolism. The crystal structure of mouse GADL1 is described, together with a solution model based on small-angle X-ray scattering data. While the overall fold and the conformation of the bound PLP are similar to those in other PLP-dependent decarboxylases, GADL1 adopts a more loose conformation in solution, which might have functional relevance in ligand binding and catalysis. The structural data raise new questions about the compactness, flexibility and conformational dynamics of PLP-dependent decarboxylases, including GADL1.

\section{Introduction}

Pyridoxal 5'-phosphate (PLP), or vitamin $\mathrm{B}_{6}$, is a versatile cofactor that is involved in many enzymatic reactions spanning multiple enzyme classes and chemical reactions (Percudani \& Peracchi, 2003). PLP-dependent decarboxylases constitute a large family of enzymes that catalyze a range of metabolic reactions. Many of these enzymes catalyze biologically well defined processes; inactivating mutations affecting them are associated with severe phenotypes, and some of them are treatment targets in human disease (Baekkeskov et al., 1990; Eliot \& Kirsch, 2004; El-Sayed \& Shindia, 2011; Paiardini et al., 2014; Sköldberg et al., 2004).

Despite extensive research, the biological functions of many PLP-dependent enzymes are still unclear. One such enzyme is glutamic acid decarboxylase-like protein 1 (GADL1), which was originally named based on its sequence homology to glutamic acid decarboxylase (GAD), which synthesizes the inhibitory neurotransmitter $\gamma$-aminobutyric acid (GABA; Fenalti et al., 2007). However, GADL1 has no reactivity towards glutamic acid and therefore is unlikely to be involved in GABA signalling (Liu et al., 2012; Winge et al., 2015). It has been suggested that GADL1 is involved in taurine production (Liu et al., 2012). On the other hand, in our recent comparative study of GADL1 and cysteine sulfinic acid decarboxylase (CSAD), an enzyme homologous to GADL1 that is involved in taurine biosynthesis, we showed that these enzymes act 
Table 1

Crystallization.

\begin{tabular}{lll}
\hline Crystal form & 1 & 2 \\
\hline Method & Sitting-drop vapour diffusion & Sitting-drop vapour diffusion \\
Plate type & Swissci 3-drop 96-well plate & Swissci 3-drop 96-well plate \\
Temperature $(\mathrm{K})$ & 293 & 281 \\
Protein concentration $\left(\mathrm{mg} \mathrm{ml}^{-1}\right)$ & 7.5 & 7 \\
Buffer composition of protein solution & $20 \mathrm{~m} M$ HEPES pH 7.4, $200 \mathrm{~m} M \mathrm{NaCl}$ & $20 \mathrm{~m} M \mathrm{HEPES} \mathrm{pH} 7.4,200 \mathrm{~m} M \mathrm{NaCl}$ \\
Composition of reservoir solution & $80 \mathrm{~m} M$ sodium cacodylate $\mathrm{pH} 6.0,14 \%$ PEG 8000, & $80 \mathrm{~m} M$ sodium cacodylate $\mathrm{pH} 7.0,13 \%$ PEG 8000, \\
& $160 \mathrm{~m} M$ calcium acetate, $15 \%$ glycerol & $160 \mathrm{~m} M$ calcium acetate, $15 \%$ glycerol \\
Volume and ratio (protein:well solution) of drop & $0.3 \mu \mathrm{l}(1: 1)$ & $1.5 \mu l(1: 2)$ \\
Volume of reservoir $(\mu \mathrm{l})$ & 40 & 40 \\
Cryoprotection solution & $75 \%(v / v)$ reservoir $+25 \%(v / v)$ PEG 200 & $80 \%(v / v)$ reservoir $+20 \%(v / v)$ glycerol \\
\hline
\end{tabular}

differently. Compared with CSAD, the activity of GADL1 towards cysteine sulfinic acid (CSA) as a substrate is much lower, and GADL1 has a stronger preference for aspartate, suggesting that GADL1 may be involved in the biosynthesis of $\beta$-alanine and its peptide derivatives, such as the neuroprotectant carnosine (Min et al., 2008; Park et al., 2014; Winge et al., 2015).

We showed in our earlier study that mouse and human brain have distinct patterns of expression of CSAD and GADL1 (Winge et al., 2015). Whereas both CSAD and GADL1 were expressed in neurons, only the CSAD mRNA was detected in astrocytes. Using a homology model of GADL1 based on the crystal structure of human CSAD ( $H s$ CSAD), we performed in silico screening of potential substrate analogues and discovered the first generation of inhibitors with partial selectivity against either GADL1 or CSAD. However, detailed mechanistic studies have been hampered by the lack of structural information.

In this study, we describe the crystal structure of mouse GADL1 (MmGADL1). Additionally, we used small-angle X-ray scattering (SAXS) to elucidate the solution shape of $M m$ GADL1. The overall fold of $M m$ GADL1 is similar to those of CSAD and other close homologues, with a flexible loop, not defined in electron density, from the apposing monomer covering the active site, which is possibly relevant in catalysis. SAXS data demonstrate that MmGADL1 adopts a loosened state in solution, which might correspond to an open conformation significant for cofactor or substrate binding.

\section{Materials and methods}

\subsection{Macromolecule production}

2.1.1. Construct preparation, protein expression and purification. The expression vector for $M m$ GADL1 was prepared in the Gateway system using pTH27 (Hammarström et al., 2006) as the destination vector. Cloning involved a twostep PCR protocol and homologous recombination into Gateway vectors using standard procedures. The resulting construct codes for an N-terminal $\mathrm{His}_{6} \mathrm{tag}$, a Tobacco etch virus (TEV) protease cleavage site and MmGADL1 residues 1-502 (UniProt entry E9QP13). A longer isoform of $M m$ GADL1 also exists (UniProtKB entry Q80WP8), and the construct corresponds to residues $49-550$ of this isoform.
2.1.2. Expression and purification of MmGADL1. $\mathrm{His}_{6}$ tagged MmGADL1 was expressed in Escherichia coli BL21CodonPlus(DE3)-RIPL cells (Stratagene) at $288 \mathrm{~K}$ using $150 \mathrm{~m} M$ IPTG induction. Cell pellets were lysed by sonication in a buffer consisting of $50 \mathrm{~m} M$ sodium phosphate buffer $\mathrm{pH}$ 7.4, $500 \mathrm{~m} M \mathrm{NaCl}, 20 \mathrm{~m} M$ imidazole, $0.2 \mathrm{mg} \mathrm{ml}^{-1}$ lysozyme, $1 \mathrm{mM} \mathrm{MgCl} 2,2 \mathrm{~m} M$ pyridoxine hydrochloride and cOmplete EDTA-free protease inhibitors (Roche). Phenylmethylsulfonyl fluoride was added to $1 \mathrm{~m} M$ immediately following sonication. The unclarified lysate was applied directly onto an IMAC HiTrap TALON crude column (GE Healthcare). The

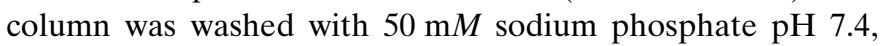
$500 \mathrm{~m} M \mathrm{NaCl}, 50 \mathrm{~m} M$ sodium phosphate $\mathrm{pH} 7.4,500 \mathrm{~m} M$ $\mathrm{NaCl}, 20 \mathrm{~m} M$ imidazole. Elution was carried out with $100 \mathrm{~m} M$ imidazole in $50 \mathrm{~m} M$ sodium phosphate $\mathrm{pH} 7.4,500 \mathrm{~m} M \mathrm{NaCl}$. Size-exclusion chromatography was performed using a Superdex HR 200 column (GE Healthcare) equilibrated with $20 \mathrm{~m} M$ HEPES, $200 \mathrm{~m} M \mathrm{NaCl} \mathrm{pH}$ 7.5.

\subsection{Crystallization}

Initial crystallization conditions for $M m$ GADL1 were obtained from the JCSG-plus screen (Molecular Dimensions) using sitting-drop vapour diffusion. The crystallization conditions, which yielded crystals with a needle morphology arranged as single crystals or point-originated clusters, were comprised of $80 \mathrm{~m} M$ sodium cacodylate $\mathrm{pH}$ 6.0-7.4, 13$14 \%(w / v)$ PEG 8000, 120-160 mM calcium acetate, 15.0$17.5 \%(w / v)$ glycerol. $0.3-1.5 \mu \mathrm{l}$ drops with different volume ratios of protein solution $\left(6.5-7.5 \mathrm{mg} \mathrm{ml}^{-1}\right.$ in $20 \mathrm{~m} M$ HEPES $\mathrm{pH} 7.4,200 \mathrm{mM} \mathrm{NaCl}$ ) and reservoir solution were used at 281 and $293 \mathrm{~K}$, equilibrating against $40 \mu \mathrm{l}$ reservoir solution. Crystals were briefly soaked in cryoprotectant solutions and flash-cooled in liquid $\mathrm{N}_{2}$. The detailed conditions used to obtain the crystals used for data collection are given in Table 1.

\subsection{Data collection, structure solution and refinement}

The $M m$ GADL1 structure was solved from the two crystal forms by molecular replacement in Phaser (McCoy et al., 2007) using the structure of $H s C S A D$ (PDB entry 2jis; Structural Genomics Consortium, unpublished work) as the search model. Crystal form 1 diffracted to $3 \AA$ resolution, while crystal form 2, which was used for initial structure solution, diffracted to $2.4 \AA$ resolution; the latter suffered from near- 
Table 2

Data collection, processing and structure refinement.

Values in parentheses are for the highest resolution shell.

\begin{tabular}{|c|c|c|}
\hline Crystal form & 1 & 2 \\
\hline \multicolumn{3}{|l|}{ Data-collection statistics } \\
\hline Wavelength $(\AA)$ & 1.0332 & 0.9763 \\
\hline Space group & $C 2$ & $P 2_{1}$ \\
\hline$a, b, c(\AA)$ & $137.4,80.6,128.5$ & $80.9,121.7,101.1$ \\
\hline$\alpha, \beta, \gamma\left({ }^{\circ}\right)$ & $90,117.9,90$ & $90,90.08,90$ \\
\hline Resolution range $(\AA)$ & $50-3.00(3.08-3.00)$ & $50-2.40(2.46-2.40)$ \\
\hline Completeness $(\%)$ & $99.4(99.5)$ & $98.7(95.9)$ \\
\hline Multiplicity & $6.4(6.0)$ & $3.6(3.1)$ \\
\hline$\langle I / \sigma(I)\rangle \dagger$ & $7.1(0.7)$ & $5.9(0.9)$ \\
\hline$R_{\text {meas }}$ & $0.328(3.535)$ & $0.188(1.466)$ \\
\hline$R_{\text {p.i.m. }}$ & $0.130(1.443)$ & $0.099(0.832)$ \\
\hline $\mathrm{CC}_{1 / 2}(\%)$ & $98.9(28.4)$ & $99.3(61.4)$ \\
\hline $\begin{array}{l}\text { Overall } B \text { factor from } \\
\text { Wilson plot }\left(\AA^{2}\right)\end{array}$ & 67 & 44 \\
\hline \multicolumn{3}{|l|}{ Refinement statistics } \\
\hline Resolution range $(\AA)$ & $50-3.0$ & - \\
\hline Final $R_{\text {cryst }}$ & 0.236 & - \\
\hline Final $R_{\text {free }}$ & 0.288 & - \\
\hline \multicolumn{3}{|l|}{ R.m.s.d.s } \\
\hline Bond lengths $(\AA)$ & 0.003 & - \\
\hline Bond angles $\left({ }^{\circ}\right)$ & 0.7 & - \\
\hline Average $B$ factor $\left(\AA^{2}\right)$ & 91.0 & - \\
\hline \multicolumn{3}{|l|}{ Ramachandran plot } \\
\hline Favoured (\%) & 92.0 & - \\
\hline Outliers (\%) & 0.6 & - \\
\hline MolProbity score [percentile] & 1.98 [99th] & - \\
\hline Twin operator/twin fraction (\%) & - & $h,-k,-l / 45$ \\
\hline $\begin{array}{l}\text { Pseudotranslation operator/ } \\
\text { fraction }(\%)\end{array}$ & - & $0.060,-0.500,0.417 / 38$ \\
\hline
\end{tabular}

$\dagger$ The mean $I / \sigma(I)$ in the outermost shell falls below 2.0 at $3.3 \AA$ for crystal form 1 and $2.7 \AA$ for crystal form 2 .

perfect pseudomerohedral twinning and high translational pseudosymmetry. Owing to these observations, both crystal forms were solved and initially refined, and the lower resolution structure, which completely lacked twinning and pseudotranslation, was considered to be better for final refinement. In essence, despite the higher nominal resolution, the twinned crystal suffering from pseudotranslation gave lower-quality electron-density maps. The twinning and pseudotranslation operations are given in Table 2 . NCS restraints were employed throughout refinement. Refinement was performed with phenix.refine (Afonine et al., 2012) and model building with Coot (Emsley \& Cowtan, 2004). The structure was validated with MolProbity (Chen et al., 2010). Data collection and refinement statistics can be found in Table 2. The resolution limits used were based on recent studies (Karplus \& Diederichs, 2015) showing that useful information is available for refinement even for data with a $\mathrm{CC}_{1 / 2}$ much lower than $50 \%$.

\subsection{Small-angle X-ray scattering}

Synchrotron SAXS data were collected on the EMBL/ DESY BioSAXS beamline P12 (Blanchet et al., 2015). The protein was at $1.6-6.5 \mathrm{mg} \mathrm{ml}^{-1}$ in $20 \mathrm{~m} M$ HEPES pH 7.4, $200 \mathrm{~m} M \mathrm{NaCl}$. The scattering data were processed and analyzed with programs from the ATSAS package (Konarev et $a l ., 2006)$. The molecular weight was determined by comparison of the forward scattering intensity, $I(0)$, with a fresh monomeric bovine serum albumin standard. Models of $M m$ GADL1 were built with GASBOR (Svergun et al., 2001) and SREFLEX (Panjkovich \& Svergun, 2016), using data extrapolated to zero concentration. Theoretical scattering curves from crystal structure coordinates were calculated with CRYSOL (Svergun et al., 1995). Model superposition was performed using SUPCOMB (Kozin \& Svergun, 2001). Details of SAXS data collection, processing and analysis are given in Table 3, and the raw SAXS scattering data are provided as Supporting Information.

\subsection{Sequence and structure analysis}

APBS (Unni et al., 2011), UCSF Chimera (Pettersen et al., 2004), PyMOL (http://www.pymol.org), ProtParam (Gasteiger et al., 2005), PDBeFold (Krissinel \& Henrick, 2004), MUSCLE (Edgar, 2004) and ESPript3.0 (Robert \& Gouet, 2014) were used for bioinformatics and structure analyses.

\section{Results and discussion}

\subsection{The crystal structure of MmGADL1}

Initial screening for crystallization conditions of $M m$ GADL1 resulted in a single condition that produced diffracting crystals. Crystals formed with a needle morphology, often growing in bunches or clusters and generally being very thin, with maximum lengths reaching $500 \mu \mathrm{m}$. The diffraction quality was initially poor, with diffraction limits of around 6-7 $\AA$ and highly smeared reflections. By optimizing the size, the amount of nucleation, and the general appearance of the crystals, the conditions given in Table 1 produced thin but nonfragile crystals that allowed structure refinement in two crystal forms to resolutions of 2.4 and $3.0 \AA$; the higher resolution data set was plagued by significant twinning and pseudotranslation (Table 2). Thus, the structure of the nontwinned crystal form is discussed here; essentially all features can also be seen in the twinned crystal.

The crystal structure revealed a single $M m$ GADL1 homodimer in the asymmetric unit, which was the expected oligomeric state based on other PLP-dependent decarboxylases (Fig. 1a). For both chains, residues 11-502 could be built, with the flexible loop common to PLP-dependent decarboxylases (Fenalti et al., 2007) being absent from the electron density (approximately residues 340-350). The overall conformation of the two chains was nearly identical (Fig. 1b).

In the crystal lattice, the protein dimers form layers in the $x y$ plane, separated by a rather uniform spacing (Fig. 1c). As the first $\sim 30$ amino acids of the tagged protein were not visible, they are most likely to occupy the space between protein dimers in the crystal. This is the likely source of the high mosaicity and incomplete lattice arrangement in the current MmGADL1 crystals.

Both active sites in the dimer are occupied by the PLP cofactor, which is covalently bound to the $\mathrm{N}^{\varepsilon}$ atom of Lys314 in each chain through a Schiff base linkage, being located at the dimer interface (Fig. 1d). Closer observation of the activesite cavity reveals that only the active imine of the linked PLP 
Table 3

Small-angle X-ray scattering.

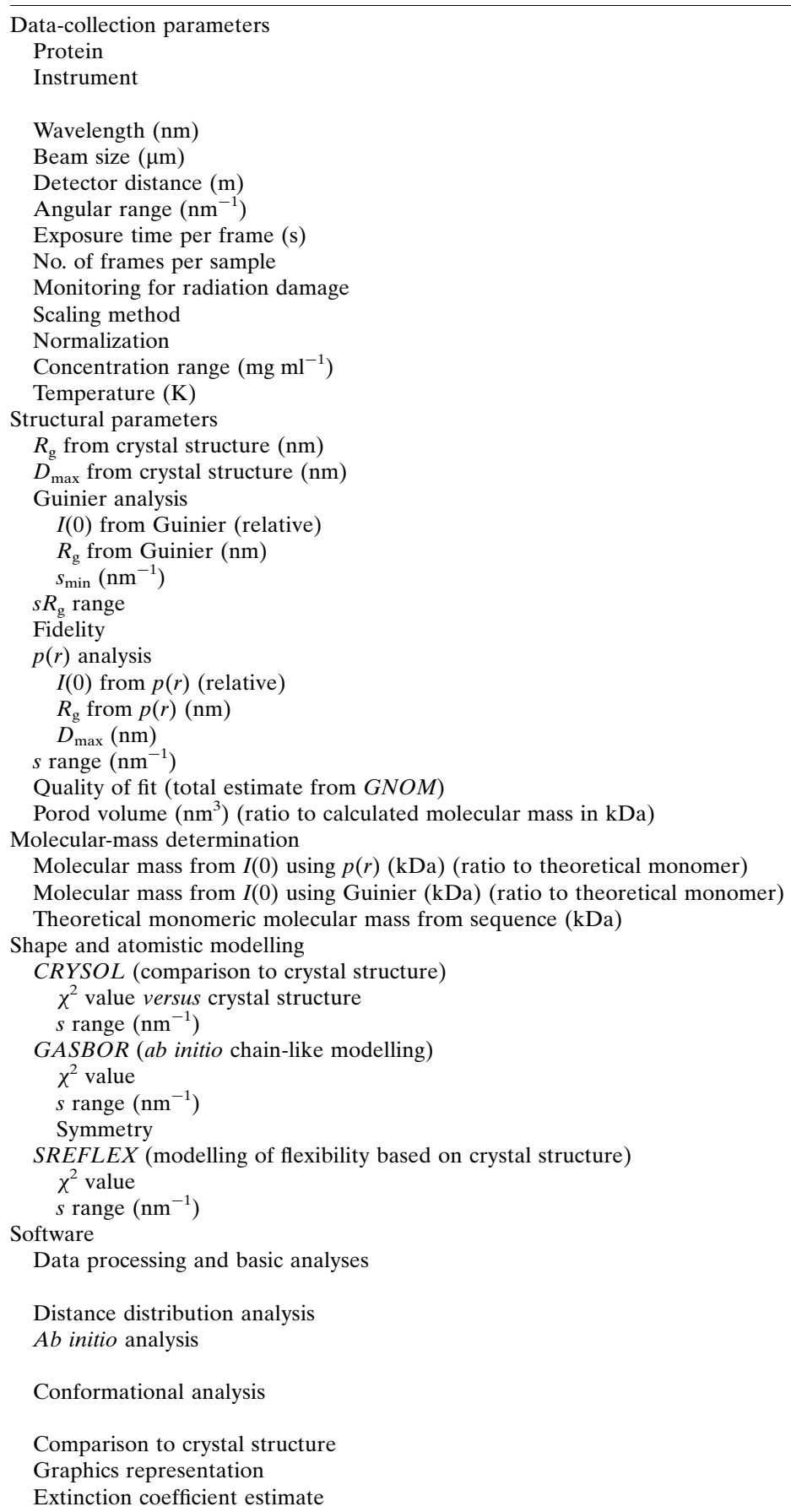

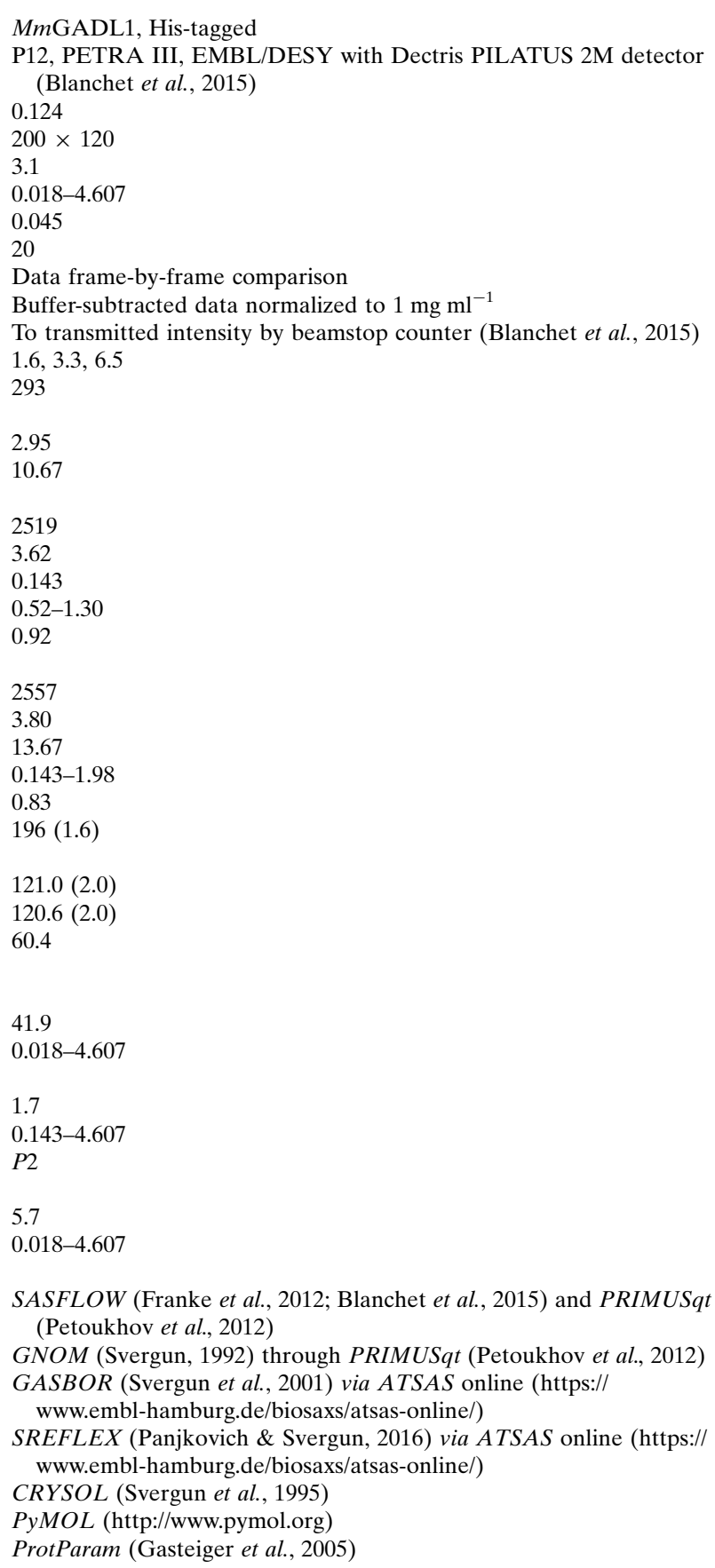

is solvent-exposed, and it resides at the end of a narrow cavity, which represents the substrate-binding pocket (Fig. 2a). Electrostatic surface analysis reveals the GADL1 active site to have a high positive charge potential (Fig. 2b). This is logical, as the substrates of GADL1 are acidic amino acids; the basic nature of the binding cavity is involved in electrostatic interactions that attract and bind the substrate, orienting it correctly for catalysis.

In our earlier work, we showed that GADL1 can use aspartate and CSA as substrates, but not glutamate (Winge et al., 2015). While the catalytic cavities of GAD and GADL1 are very similar, it is currently difficult to pinpoint which features of the active site might be responsible for selectivity between such similar substrates. High-resolution structures of GADL1 and its closest homologues with bound active-site ligands will clearly be required. Importantly, a large part of the active-site cavity wall will be formed by the flexible loop in the substrate-bound state; the flexible loop is invisible in most PLP-dependent decarboxylase crystal structures, but harbours a Tyr residue that is likely to be important for catalysis. 

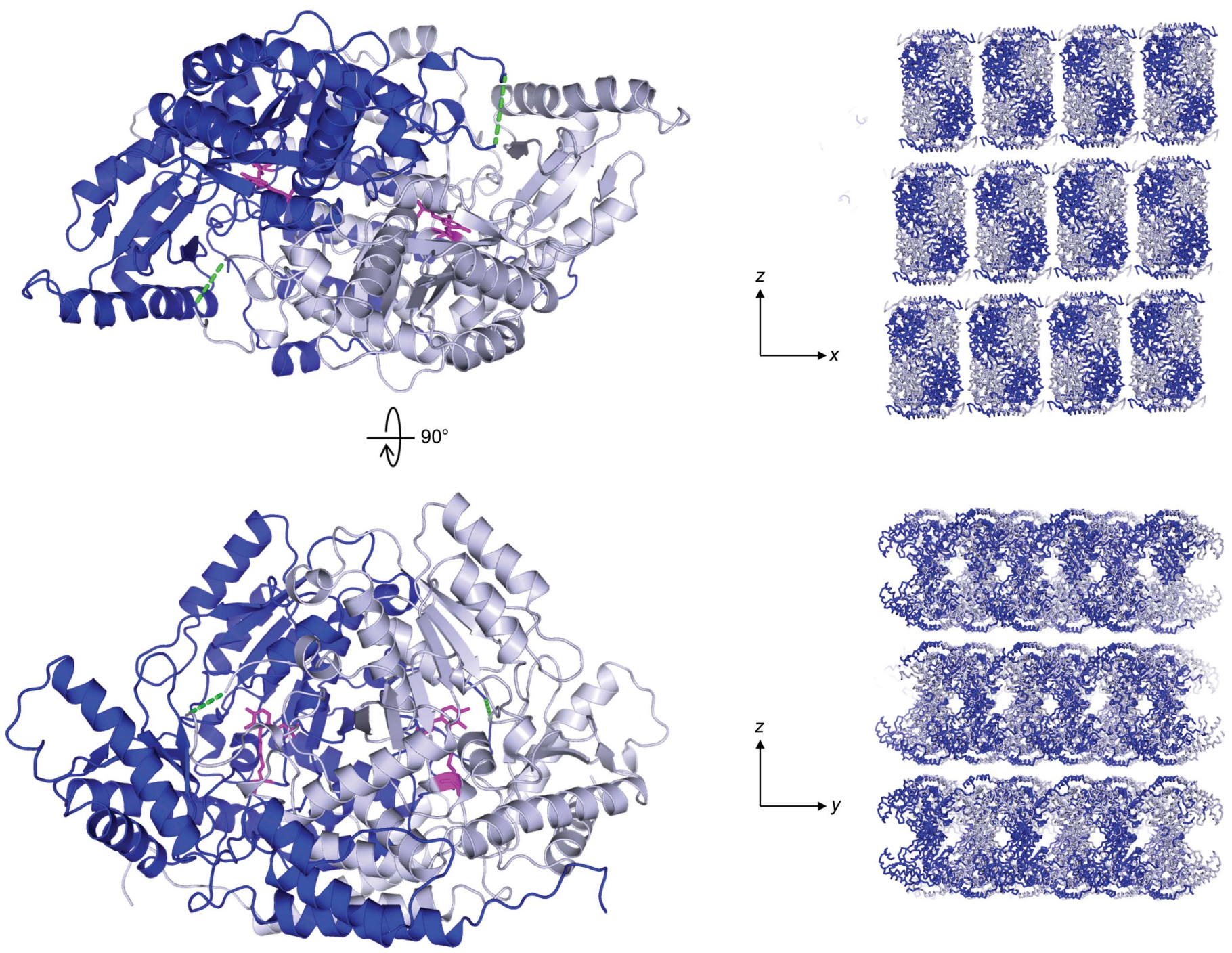

(a)

(c)

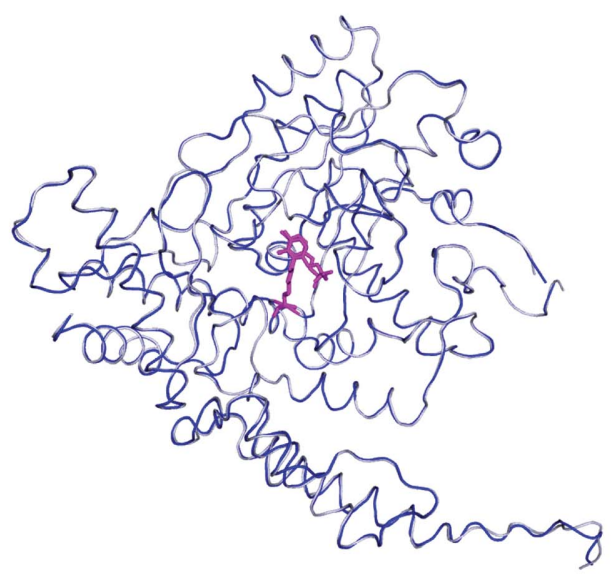

(b)

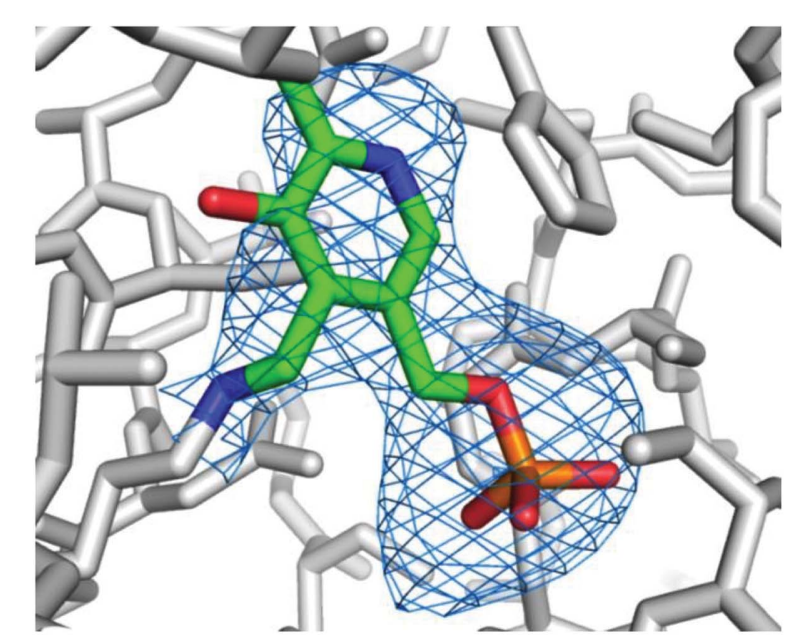

$(d)$

Figure 1

Overall structure of MmGADL1. (a) The MmGADL1 dimer. The PLP molecule covalently bound to Lys314 is shown in magenta. The green dashed line indicates the position of the flexible loop covering the active site. (b) Superposition of the two MmGADL1 monomers in the homodimer. The covalently bound PLP is shown in magenta. The two monomers are essentially identical. (c) Crystal lattice arrangement of MmGADL1 in two different planes. Note the uniform $11 \AA$ cavities in the $x y$ plane between protein layers. (d) Omit $F_{\mathrm{o}}-F_{\mathrm{c}}$ difference map (blue mesh) contoured at $2 \sigma$ for the covalently bound PLP cofactor on Lys314. 


\subsection{MmGADL1 adopts an open conformation in solution}

We used SAXS to validate the crystal structure and to determine the conformation of MmGADL1 in solution (Fig. 3, Table 3). As is apparent from the scattering data and the dimensionless Kratky plot, GADL1 presents a folded shape. While the molecular mass calculated from the forward scattering intensity accurately matches that of a dimer, the theoretical scattering curve calculated from the crystal structure differs significantly. The shape in solution is more elongated than in the crystal. The radii of gyration between the theoretical scattering curve from the crystal structure and the experimental value from Guinier analysis differ by nearly $1 \mathrm{~nm}$, indicating a large difference in conformation. The maximum diameter is $3 \mathrm{~nm}$ larger in solution than in the crystal state.

The GASBOR chain-like dummy residue model of $M m$ GADL1 is elongated compared with the crystal structure (Fig. 3e). Attempts to model the missing N-terminal portion, while keeping the dimeric crystal structure fixed, did not fit the experimental SAXS data well (data not shown). We thus employed the recently described SREFLEX method (Panjkovich \& Svergun, 2016) to take advantage of normal- mode analysis of the crystal structure in SAXS modelling. The SREFLEX model fits the scattering data well and suggests a clearly loosened solution conformation (Fig. $3 f$ ), in contrast to the compact globular structures observed for PLP-dependent decarboxylases in the crystalline state. The open conformation is not similar to the open conformation observed for DOPA decarboxylase in the crystalline state (Giardina et al., 2011; Fig. $3 g$ ), in which case the opening occurs in the centre of the dimer. The observed open-close movement is likely to be of functional relevance in the GADL1 catalytic cycle. Whether the different conformations are linked to the binding of ligands remains to be studied. While our GADL1 preparation is enzymatically active (Winge et al., 2015), and the crystal is apparently fully occupied with covalently bound PLP, we cannot currently rule out the presence of PLP-deficient GADL1 in the purified SAXS sample, since crystallization might have enriched a cofactor-bound conformation of the protein.

\subsection{Comparison to other PLP-dependent decarboxylases}

While $M m$ GADL1 and its homologues show low sequence conservation, apart from a few fully conserved core elements

Table 4

Structural homologues of MmGADL1.

The homologues were detected by an SSM analysis using PDBeFold.

\begin{tabular}{|c|c|c|c|c|c|c|c|c|c|}
\hline Protein & Organism & $\begin{array}{l}\text { PDB } \\
\text { entry }\end{array}$ & Reference & Chain & $Q$-score & $\begin{array}{l}\text { R.m.s.d. } \\
(\AA)\end{array}$ & $\begin{array}{l}\text { Sequence } \\
\text { identity }(\%)\end{array}$ & $\begin{array}{l}\text { UniProtKB } \\
\text { entry }\end{array}$ & $\begin{array}{l}\text { Aligned } \\
\text { residues }\end{array}$ \\
\hline$M m$ GADL1 & Mus musculus & 6enz & This study & $A$ & - & - & - & E9QP13 & $1-502$ \\
\hline$H s \mathrm{CSAD}$ & Homo sapiens & $2 \mathrm{jis}$ & Unpublished work & $B$ & 0.586 & 0.85 & 62.0 & Q9Y600 & $1-493$ \\
\hline$H s \mathrm{GAD} 65$ & Homo sapiens & 2okk & Fenalti et al. (2007) & $A$ & 0.565 & 1.06 & 49.4 & Q05329 & $88-584$ \\
\hline HsGAD67 & Homo sapiens & 2okj & Fenalti et al. (2007) & $B$ & 0.532 & 1.15 & 50.9 & Q9925 & $93-594$ \\
\hline$S t \mathrm{PDD}$ & Sphaerobacter thermophilus & 4rit & Unpublished work & $B$ & 0.461 & 1.96 & 28.9 & D1C7D8 & $1-483$ \\
\hline RUMGNA_01526 & Ruminococcus gnavus & 4obu & Williams et al. (2014) & $H$ & 0.492 & 1.79 & 25.6 & A7B1V0 & $1-490$ \\
\hline$V p \mathrm{GAD}$ & Vibrio parahaemolyticus & $2 \mathrm{qma}$ & Unpublished work & $B$ & 0.442 & 2.16 & 25.8 & Q87NC6 & 464-957 \\
\hline$H s \mathrm{HDC}$ & Homo sapiens & $4 \mathrm{e} 1 \mathrm{o}$ & Komori et al. (2012) & $C$ & 0.425 & 2.13 & 26.1 & P19113 & $2-477$ \\
\hline$H s \mathrm{DDC}$ & Homo sapiens & $3 \mathrm{rbl}$ & Giardina et al. (2011) & $A$ & 0.397 & 2.61 & 23.3 & P20711 & $1-480$ \\
\hline
\end{tabular}

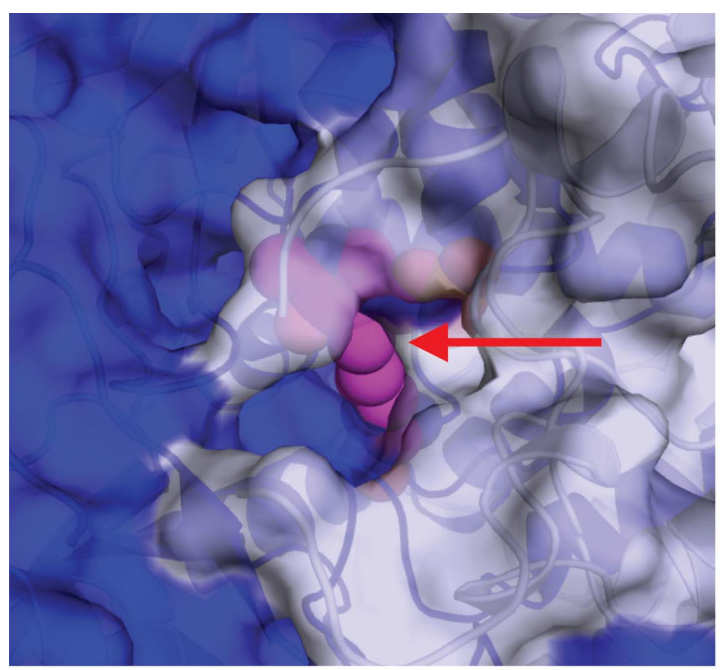

(a)

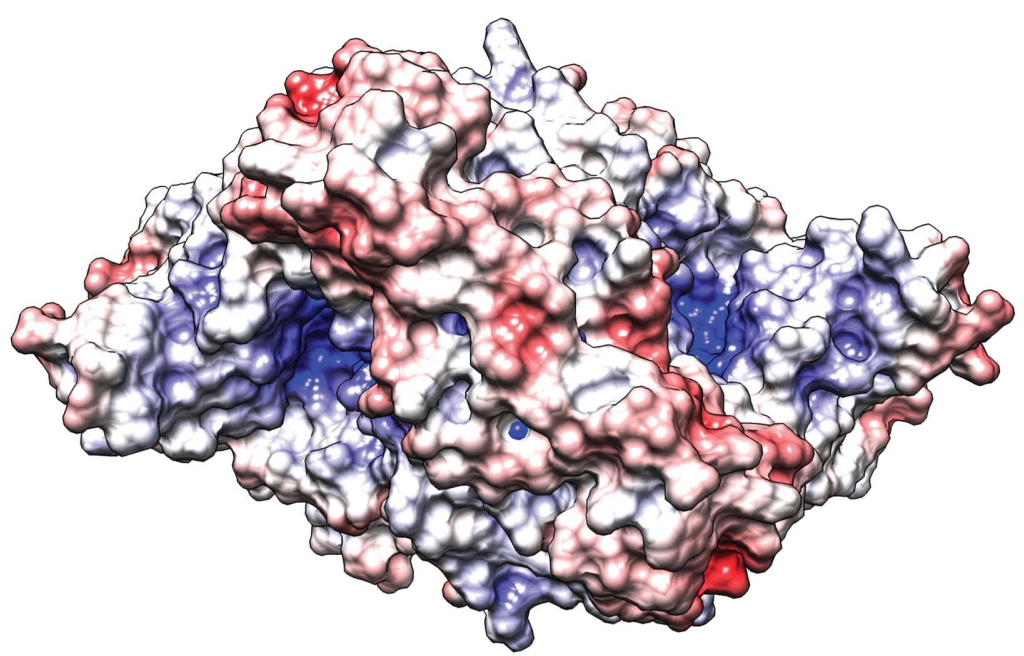

(b)

Figure 2

The MmGADL1 active site. (a) Close-up view of the active-site cavity with the reactive moiety of PLP (magenta) visible (red arrow). Note how the cofactor lies at the interface between two protein monomers (blue and grey). (b) Surface electrostatics of MmGADL1. The positively charged cavity corresponds to the active site (blue). 
around the active site (Fig. 4a), comparison of the structures of MmGADL1 and its homologues reveals a conserved structural fold with little variance in the arrangement of the PLPlinked Lys residue (Figs. $4 b$ and $4 c$, Table 4). The sequence conservation between MmGADL1, $H s$ CSAD and $H s$ GADs (Fenalti et al., 2007) is higher than those with human histidine decarboxylase (HDC; Komori et al., 2012) and DOPA decarboxylase (DDC; Giardina et al., 2011; Winge et al., 2015). The latter present similar levels of sequence homology to GADL1 as the bacterial enzymes Sphaerobacter thermophilus PLPdependent decarboxylase (StPDD), Vibrio parahaemolyticus GAD (VpGAD) and the tryptamine-synthesizing enzyme from the gut bacterium Ruminococcus gnavus (RUMGNA_ 01526; Williams et al., 2014). Despite low sequence homology, the $R$. gnavus enzyme displays high structural similarity to GADL1, suggesting conservation of the fold of PLP- dependent decarboxylases involved in neurotransmitter synthesis. It is interesting to note that the absence of PLP in the active site neither alters the overall tightness of the superposed proteins nor changes the position of the conserved Lys in most structures. In the future, experimental solutionstate methods, such as SAXS, may help to distinguish functionally relevant conformational states from possible crystallographic artifacts. These observations conflict with earlier results described for $H s \mathrm{DDC}$ in the beginning of its PLP accumulation-dependent conformational landscape, where a more open conformation was evident in the crystalline state with the active-site Lys residue oriented away from the PLPbinding pocket (Giardina et al., 2011).

The substrate specificity and physiological function of GADL1 remain enigmatic. However, the conserved structural details and distinct expression patterns of GADL1 suggest

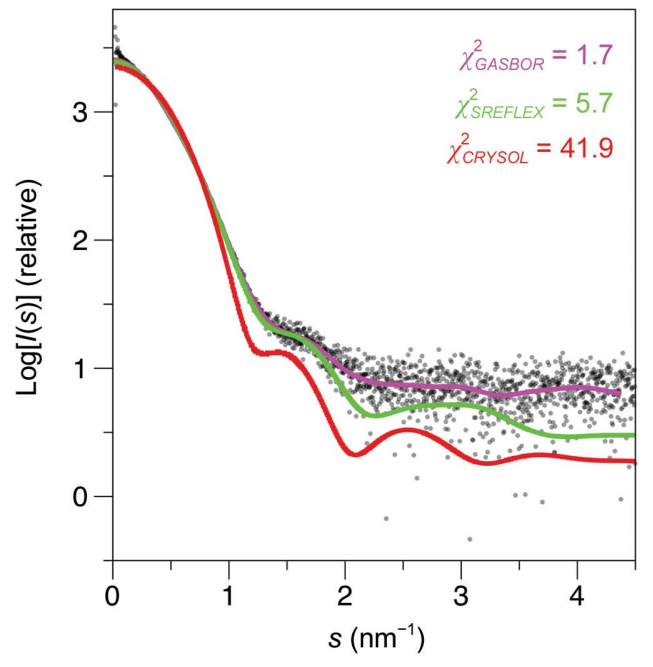

(a)

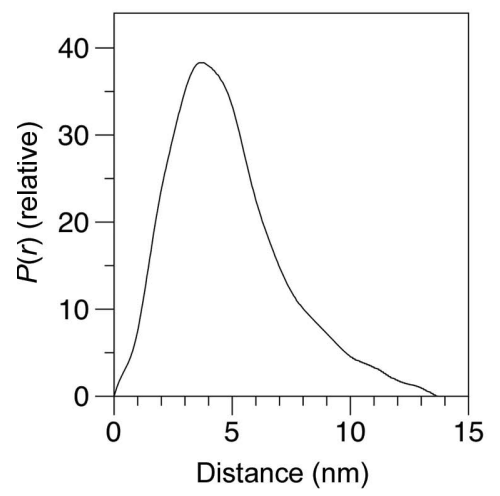

(d)

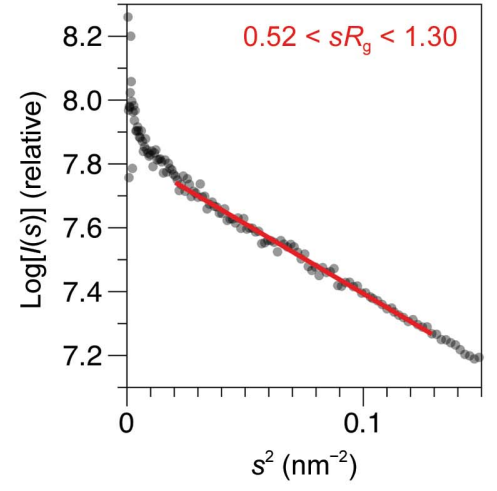

(b)

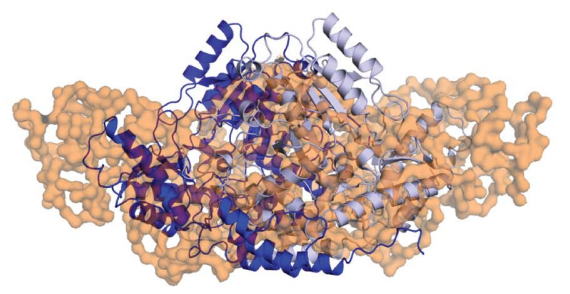

(e)

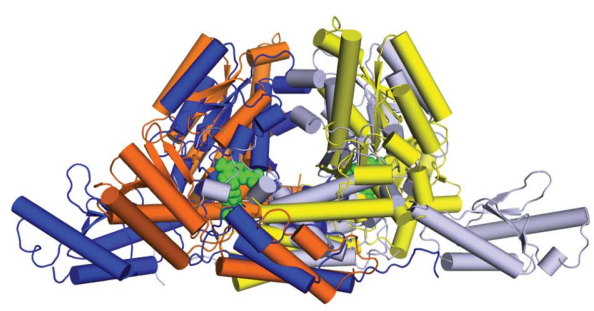

(g)

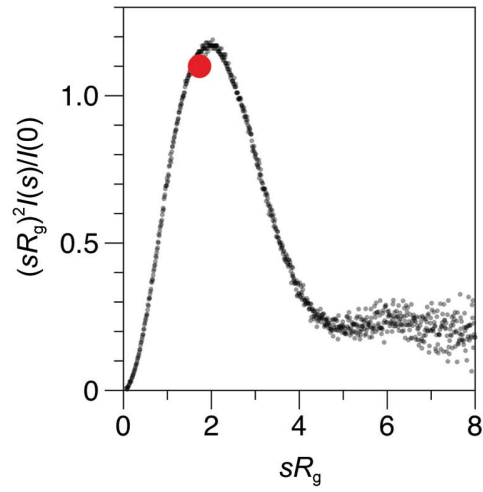

(c)

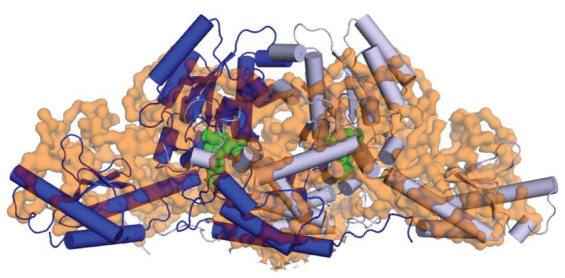

$\frac{\gamma}{\gamma} 90^{\circ}$

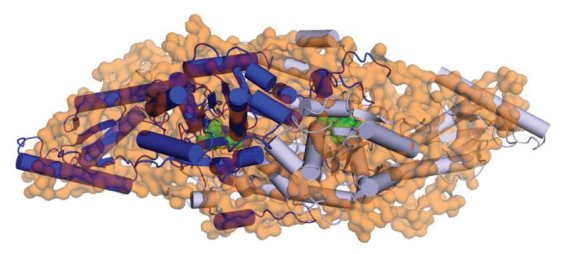

(f)

Figure 3

Structure of MmGADL1 in solution determined by SAXS. (a) Raw SAXS data (dots) overlaid with GASBOR (pink) and SREFLEX (green) model fits, as well as the theoretical scattering curve calculated from the crystal structure using CRYSOL (red). (b) Guinier plot. (c) The dimensionless Kratky plot indicates that $M m$ GADL1 is folded, with limited flexibility. The red dot indicates the theoretical position of the peak in a folded globular protein. (d) Distance distribution of MmGADL1. (e) The GASBOR model (orange) superimposed with the crystal structure of MmGADL1 indicates a much more elongated conformation in solution. ( $f$ ) Superposition of the SREFLEX (blue/grey) and GASBOR (orange) models suggests conformational changes relative to the crystal structure. ( $g$ ) Comparison of the 'open' conformation of DDC (orange/yellow; Giardina et al., 2011) and the open conformation of $M m$ GADL1 (blue/grey) seen here in solution. 
that it plays a specific physiological role. Variants of GADL1 have been linked to lithium response in bipolar disorder patients (Chen et al., 2014), suggesting a role for GADL1 in lithium pharmacodynamics or kinetics. However, these findings have not been replicated by others, and they have been subject to much controversy (Birnbaum et al., 2014; Cruceanu et al., 2015; Kotambail et al., 2015; Winge et al., 2015; Chen et al., 2016).

Owing to their common mechanistic features, many PLPdependent enzymes are able to catalyze multiple biochemical reactions, making it difficult to define their primary biological function (Percudani \& Peracchi, 2003). Of the known GADL1 substrates, Asp appears as the most characteristic substrate for GADL1 (Winge et al., 2015), although the $K_{\mathrm{m}}$ is relatively high and the catalytic efficiency is low. Nevertheless, the $K_{\mathrm{m}}$ of GADL1 for Asp is in the physiological range, and one could speculate that GADL1 is involved in sensing selected metabolite levels. Relatively low binding affinity is a hallmark of many proteins with signalling roles, which have evolved as sensors of ligand availability; such proteins include, for example, the calcium sensors calmodulin and annexin (Kursula, 2014; Monastyrskaya et al., 2007). Conformational flexibility, as observed here for GADL1 in solution, might have relevance in such a function.
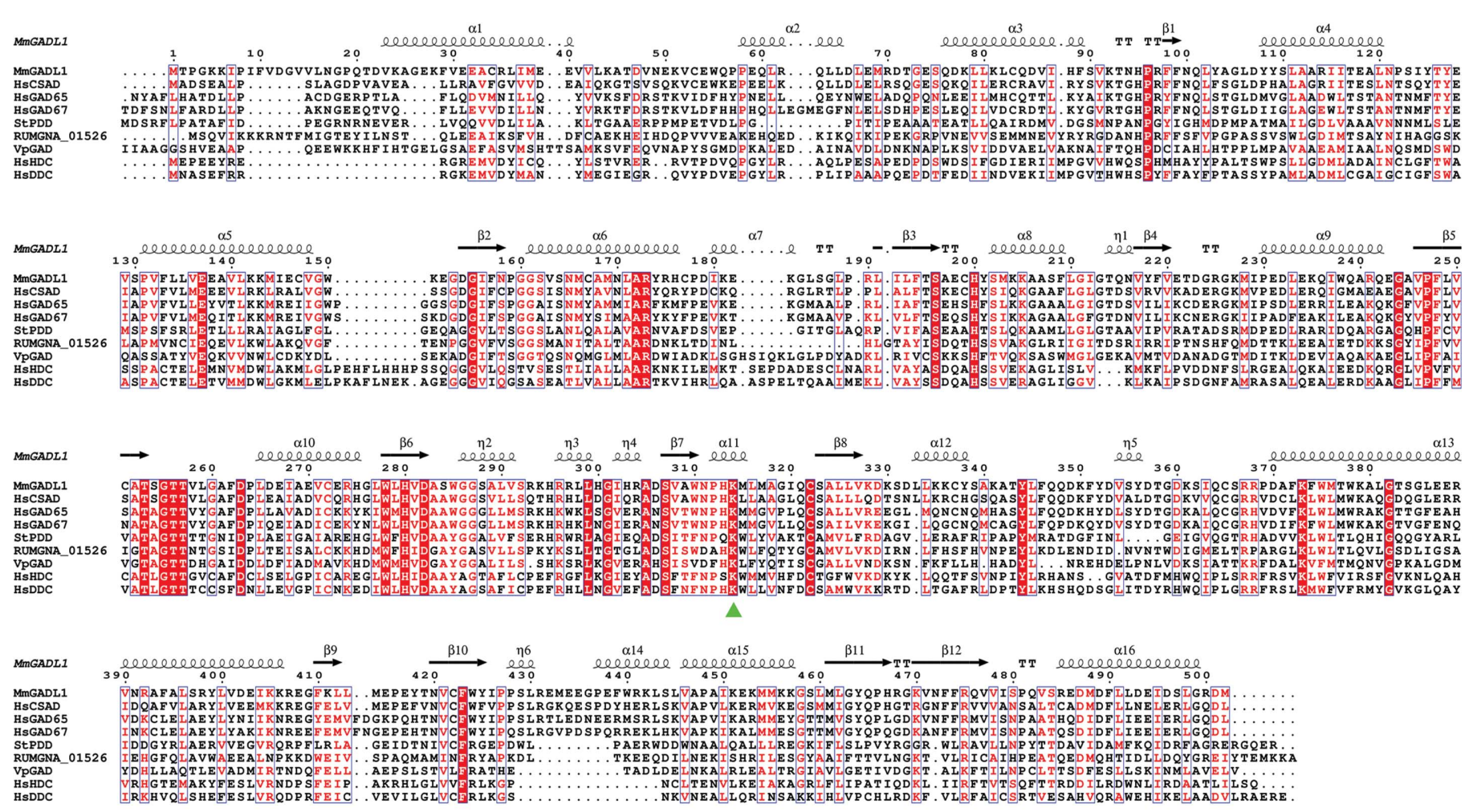

(a)

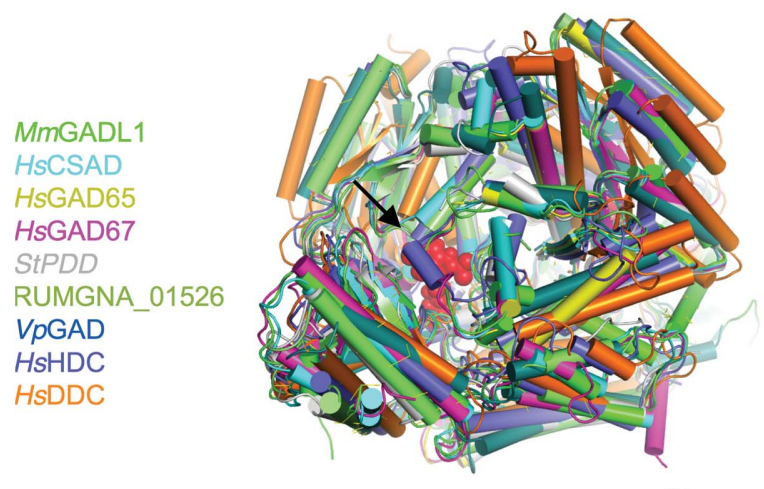

(b)

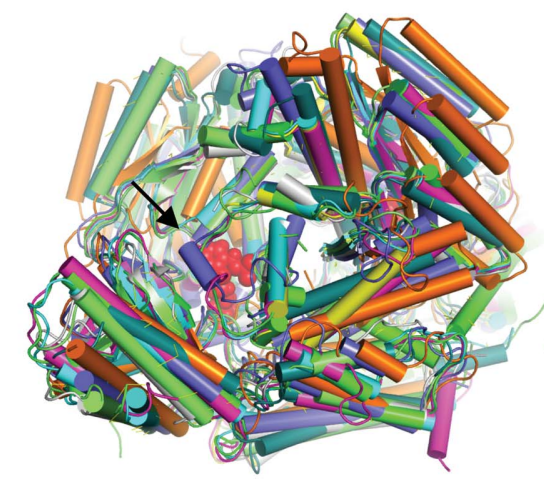

$(c)$

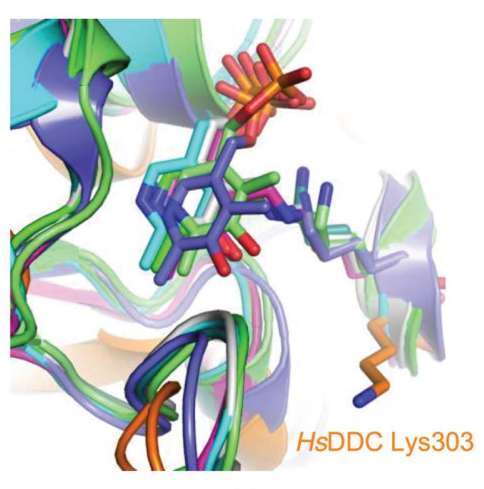

Figure 4

Comparison of MmGADL1 with other PLP-dependent decarboxylases. (a) Sequence alignment of MmGADL1 with homologous structures. The conserved PLP-modified lysine is indicated (green triangle). Secondary structure elements and sequence numbering correspond to MmGADL1. (b) Stereo image of a structural superposition of PLP-dependent decarboxylase homologues, viewed towards the active-site cavity. The covalently linked $M m$ GADL1 PLP moiety is depicted as red spheres. The black arrow shows the position of the active-site-covering loop, which is disordered in the $M m$ GADL1 crystal structure. (c) Conservation of PLP conformation in the superposed PLP-dependent decarboxylase structures. 


\section{Concluding remarks}

The physiological functions and enzymatic properties of GADL1 are subject to further studies. The structure of $M m$ GADL1 and its flexibility in solution, coupled to structural conservation with other PLP-dependent enzymes, point towards functional relevance of these features within the enzyme family. Important future work will concentrate on high-resolution structural details of substrate and inhibitor binding by GADL1, and on comparing these with those of CSAD, GAD and other PLP-dependent decarboxylases. Crucial topics to address will include the fine details of substrate specificity determinants in PLP-dependent decarboxylases, as well as the relevance of the conformational changes observed here to the catalytic cycle of this family of enzymes.

\section{Acknowledgements}

We gratefully acknowledge access to the synchrotronradiation facilities and the outstanding beamline support at EMBL/DESY, Hamburg, Germany.

\section{Funding information}

Funding for this research was provided by: Helse Vest (grant to Jan Haavik); Norges Forskningsråd (grant to Petri Kursula); Sigrid Juséliuksen Säätiö (grant to Petri Kursula); Stiftelsen Kristian Gerhard Jebsen (grant to Jan Haavik); Seventh Framework Programme (grant No. 602805 to Jan Haavik).

\section{References}

Afonine, P. V., Grosse-Kunstleve, R. W., Echols, N., Headd, J. J., Moriarty, N. W., Mustyakimov, M., Terwilliger, T. C., Urzhumtsev, A., Zwart, P. H. \& Adams, P. D. (2012). Acta Cryst. D68, 352-367.

Baekkeskov, S., Aanstoot, H.-J., Christgau, S., Reetz, A., Solimena, M., Cascalho, M., Folli, F., Richter-Olesen, H. \& Camilli, P.-D. (1990). Nature (London), 347, 151-156.

Birnbaum, R., Shin, J. H. \& Weinberger, D. (2014). N. Engl. J. Med. 370, 1855-1856.

Blanchet, C. E., Spilotros, A., Schwemmer, F., Graewert, M. A., Kikhney, A., Jeffries, C. M., Franke, D., Mark, D., Zengerle, R., Cipriani, F., Fiedler, S., Roessle, M. \& Svergun, D. I. (2015). J. Appl. Cryst. 48, 431-443.

Chen, C.-H. et al. (2014). N. Engl. J. Med. 370, 119-128.

Chen, C.-K., Lee, C.-S., Chen, H.-Y., Wu, L. S.-H., Chang, J.-C., Liu, C.-Y. \& Cheng, A. T.-A. (2016). BJPsych Open, 2, 301-306.

Chen, V. B., Arendall, W. B., Headd, J. J., Keedy, D. A., Immormino, R. M., Kapral, G. J., Murray, L. W., Richardson, J. S. \& Richardson, D. C. (2010). Acta Cryst. D66, 12-21.

Cruceanu, C., Alda, M., Dion, P. A., Turecki, G. \& Rouleau, G. A. (2015). Am. J. Psychiatry, 172, 94-95.

Edgar, R. C. (2004). Nucleic Acids Res. 32, 1792-1797.

Eliot, A. \& Kirsch, J. (2004). Annu. Rev. Biochem. 73, 383-415.

El-Sayed, A. S. \& Shindia, A. A. (2011). Targets in Gene Therapy, edited by Y. You, ch. 7. Rijeka: Intech. https://doi.org/10.5772/ 17449.

Emsley, P. \& Cowtan, K. (2004). Acta Cryst. D60, 2126-2132.

Fenalti, G. et al. (2007). Nature Struct. Mol. Biol. 14, 280-286.
Franke, D., Kikhney, A. G. \& Svergun, D. I. (2012). Nucl. Instrum. Methods Phys. Res. A, 689, 52-59.

Gasteiger, E., Hoogland, C., Gattiker, A., Duvaud, S., Wilkins, M. R., Appel, R. D. \& Bairoch, A. (2005). The Proteomics Protocols Handbook, edited by J. M. Walker, pp. 571-607. Totowa: Humana Press.

Giardina, G., Montioli, R., Gianni, S., Cellini, B., Paiardini, A., Voltattorni, C. B. \& Cutruzzolà, F. (2011). Proc. Natl Acad. Sci. USA, 108, 20514-20519.

Hammarström, M., Woestenenk, E., Hellgren, N., Härd, T. \& Berglund, H. (2006). J. Struct. Funct. Genomics, 7, 1-14.

Karplus, P. A. \& Diederichs, K. (2015). Curr. Opin. Struct. Biol. 34, 60-68.

Komori, H., Nitta, Y., Ueno, H. \& Higuchi, Y. (2012). J. Biol. Chem. 287, 29175-29183.

Konarev, P. V., Petoukhov, M. V., Volkov, V. V. \& Svergun, D. I. (2006). J. Appl. Cryst. 39, 277-286.

Kotambail, A., Mathur, A., Bhat, S. M., Rai, P. S., Sharma, P. S. \& Satyamoorthy, K. (2015). Psychiatr. Genet. 25, 39-40.

Kozin, M. B. \& Svergun, D. I. (2001). J. Appl. Cryst. 34, 33-41.

Krissinel, E. \& Henrick, K. (2004). Acta Cryst. D60, 2256-2268.

Kursula, P. (2014). Amino Acids, 46, 2295-2304.

Liu, P., Ge, X., Ding, H., Jiang, H., Christensen, B. M. \& Li, J. (2012). J. Biol. Chem. 287, 40898-40906.

McCoy, A. J., Grosse-Kunstleve, R. W., Adams, P. D., Winn, M. D., Storoni, L. C. \& Read, R. J. (2007). J. Appl. Cryst. 40, 658-674.

Min, J., Senut, M., Rajanikant, K., Greenberg, E., Bandagi, R., Zemke, D., Mousa, A., Kassab, M., Farooq, M. U., Gupta, R. \& Majid, A. (2008). J. Neurosci. Res. 86, 2984-2991.

Monastyrskaya, K., Babiychuk, E. B., Hostettler, A., Rescher, U. \& Draeger, A. (2007). Cell Calcium, 41, 207-219.

Paiardini, A., Contestabile, R., Buckle, A. M. \& Cellini, B. (2014). Biomed. Res. Int. 2014, 856076.

Panjkovich, A. \& Svergun, D. I. (2016). Phys. Chem. Chem. Phys. 18, 5707-5719.

Park, H., Han, K., Shin, J., Park, J., Song, K. \& Kim, D. (2014). J. Korean. Neurosurg. Soc. 55, 125-130.

Percudani, R. \& Peracchi, A. (2003). EMBO Rep. 4, 850-854.

Petoukhov, M. V., Franke, D., Shkumatov, A. V., Tria, G., Kikhney, A. G., Gajda, M., Gorba, C., Mertens, H. D. T., Konarev, P. V. \& Svergun, D. I. (2012). J. Appl. Cryst. 45, 342-350.

Pettersen, E. F., Goddard, T. D., Huang, C. C., Couch, G. S., Greenblatt, D. M., Meng, E. C. \& Ferrin, T. E. (2004). J. Comput. Chem. 25, 1605-1612.

Robert, X. \& Gouet, P. (2014). Nucleic Acids Res. 42, W320W324.

Sköldberg, F., Rorsman, F., Perheentupa, J., Landin-Olsson, M., Husebye, E., Gustafsson, J. \& Kämpe, O. (2004). J. Clin. Endocrinol. Metab. 89, 1636-1640.

Svergun, D. I. (1992). J. Appl. Cryst. 25, 495-503.

Svergun, D., Barberato, C. \& Koch, M. H. J. (1995). J. Appl. Cryst. 28, 768-773.

Svergun, D. I., Petoukhov, M. V. \& Koch, M. H. J. (2001). Biophys. J. 80, 2946-2953.

Unni, S., Huang, Y., Hanson, R., Tobias, M., Krishnan, S., Li, W. W., Nielsen, J. E. \& Baker, N. A. (2011). J. Comput. Chem. 32, 14881491.

Williams, B. B., Van Benschoten, A. H., Cimermancic, P., Donia, M. S., Zimmermann, M., Taketani, M., Ishihara, A., Kashyap, P. C., Fraser, J. S. \& Fischbach, M. A. (2014). Cell Host Microbe, 16, 495503.

Winge, I., Teigen, K., Fossbakk, A., Mahootchi, E., Kleppe, R., Sköldberg, F., Kämpe, O. \& Haavik, J. (2015). Neurochem. Int. 90, 173-184. 